\title{
EFFECT OF THE NEW EQUINOX DEFINITION ON THE ZERO-POINT OF LONGITUDE OF THE INDIAN CALENDAR
}

\author{
A.K. Bhatnagar \\ Positional Astronomy Centre \\ P 546, Block N, 1st Floor \\ New Alipore, 700053 Calcutta, India
}

ABSTRACT. Indian calendars follow a sidereal system of astronomy taking a fixed initial point on the ecliptic as the origin from which the longitudes are measured. Its position for the official Indian Calendar has been defined by the Calendar Reform Committee (1955) as the point on the ecliptic whose true tropical longitude was $23^{\circ} 15^{\prime} 00^{\prime \prime}$ as on 21 March $1956,0^{\text {h }}$ UT. Its position was determined upto the year 1984 in accordance with Newcomb's value for general precession using the relation

$$
\mathrm{A}=22^{\circ} 27^{\prime} 37.65+5025.75 \mathrm{~T}+1 \text { ". }^{\prime \prime} 1 \mathrm{~T}^{2}
$$

where $\mathrm{T}$ is in centuries of 36525 ephemeris days from 1900 January $0.5 \mathrm{ET}$. Recent changes in the location and the motion of the equinox with reference to the epoch $\mathrm{J} 2000.0$ have necessitated corresponding changes to be included in the determination of the mean and true positions of the above initial point. The new algorithm worked out is

$\mathrm{A}=23^{\circ} 51^{\prime} 25.532$ + 5029."0966 T + 1."11161 $\mathrm{T}^{2}$

where $\mathrm{T}$ is in Julian centuries of 36525 days from $\mathrm{J} 2000.0$.

\section{THE CONSTRUCTION OF A FRAME OF HOMOGENEOUS ACCURACY BASED ON SPACE OBSERVATIONS}

\author{
B.I. VlasOV \\ VNIIFTRI \\ Institute for Physical, Technical and Radio Measurements \\ 141570 Moscow, USSR
}

ABSTRACT. A definition of the initium-method of spherical arcs is proposed. Theoretical and operational aspects of the method are discussed. Geometrical homogeneity is achieved by measuring arc distances between vertices of the octant (a right-angle spherical triangle) and the object in question. The measurements are made with the use of the standard angle $\gamma$ on board a satellite with rotation rate reconstructions with splines. The chosen standard angle is discussed. The accuracy estimations are given. 